\title{
keyplayer: An R Package for Locating Key Players in Social Networks
}

by Weihua An and Yu-Hsin Liu

\begin{abstract}
Interest in social network analysis has exploded in the past few years, partly thanks to the advancements in statistical methods and computing for network analysis. A wide range of the methods for network analysis is already covered by existent $\mathrm{R}$ packages. However, no comprehensive packages are available to calculate group centrality scores and to identify key players (i.e., those players who constitute the most central group) in a network. These functionalities are important because, for example, many social and health interventions rely on key players to facilitate the intervention. Identifying key players is challenging because players who are individually the most central are not necessarily the most central as a group due to redundancy in their connections. In this paper we develop methods and tools for computing group centrality scores and for identifying key players in social networks. We illustrate the methods using both simulated and empirical examples. The package keyplayer providing the presented methods is available from Comprehensive R Archive Network (CRAN).
\end{abstract}

\section{Introduction}

Interest in social network analysis has grown rapidly in the past few years. This was due partly to the advancements in statistical methods and computing for network analysis and partly to the increasing availability of social network data (e.g., network data generated by social media). A wide range of the methods for network analysis is already covered by R packages such as network (Butts, 2008b), sna (Butts, 2008a), igraph (Csardi and Nepusz, 2006), statnet (Handcock et al., 2008), RSiena (Ripley et al., 2013), etc. However, none of these packages provides a comprehensive toolbox to calculate group centrality measures and to identify key players, who constitute the most central group, in a network. Determining the key players in a network is very important because many social and health interventions rely on key players to facilitate the intervention. For example, Kelly et al. (1991) and Latkin (1998) trained peer leaders as educators to promote HIV prevention. Campbell et al. (2008) and An (2015) used peer leaders to facilitate smoking prevention. Borgatti (2006) and Ressler (2006) suggested removing key figures among terrorists to most widely disrupt terrorism. More examples of this sort can be found in Valente and Pumpuang (2007), Banerjee et al. (2013), etc. Identifying key players is challenging because players who are individually the most central are not necessarily the most central as a group due to redundancy in their connections. In a seminal paper, Borgatti (2006) pointed out the problem and proposed methods for identifying key players in social networks.

To the best of our knowledge, the keyplayer function in UCINET (Borgatti et al., 2002) is the first implementation of the methods detailed in Borgatti (2006). It has evolved from a separate add-on to UCINET to a built-in function UCINET. In this paper, we present the keyplayer package (An and Liu, 2016) in R, which differs from the keyplayer function in UCINET in several aspects. (1) Unlike the keyplayer function in UCINET which is only applicable to binary networks, keyplayer in R can be used for both binary and weighted networks. (2) The keyplayer package includes more centrality measures for choosing key players than what is currently available in the keyplayer function in UCINET. (3) keyplayer provides better integration with other open-source packages in R. Overall, the keyplayer function in UCINET is useful for researchers who are more familiar with UCINET and would like to utilize other functionalities provided by UCINET, whereas keyplayer is designed for users who are more familiar with $\mathrm{R}$ and who plan to do more computational work.

The influenceR package (Simon and Aditya, 2015) aims to provide calculations of several node centrality measures that were previously unavailable in other packages, such as the constraint index (Burt, 1992) and the bridging score (Valente and Fujimoto, 2010). It can also be used to identify key players in a network. But in comparison to keyplayer, it utilizes only one centrality metric when selecting key players whereas keyplayer includes eight different metrics. Also, influenceR currently works only for undirected networks whereas keyplayer works for both undirected and directed networks. Both packages provide parallel computation. influenceR relies on OpenMP for parallel computation whereas keyplayer utilizes the base package parallel which is readily available in $R$. Last, influenceR focuses on computing centrality measures at the node level whereas keyplayer is more interested in providing centrality measures at the group level. Overall, keyplayer provides more comprehensive functionalities for calculating group centrality measures and for selecting key players.

The algorithm for identifying key players in package keyplayer essentially consists of three steps. First, users choose a metric to measure centrality in a network. Second, the algorithm (specifically the 
$W=\left[\begin{array}{lllll}0 & 1 & 3 & 0 & 0 \\ 0 & 0 & 0 & 4 & 0 \\ 1 & 1 & 0 & 2 & 0 \\ 0 & 0 & 0 & 0 & 3 \\ 0 & 2 & 0 & 0 & 0\end{array}\right]$

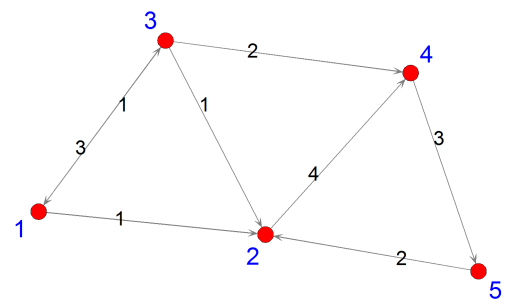

Figure 1: An adjacency matrix (left) and the corresponding network graph (right).

kpcent function) will randomly pick a group of players and measure their group centrality. Third, the algorithm (specifically the kpset function) will select the group of players with the highest group centrality as the desired key players. In general, users only need to employ the kpset function by specifying a centrality metric and the number of key players to be selected. The function will return a set of players who are the most central as a group. We also make the auxiliary function kpcent available. If users specify a centrality metric and the indices of a group of players, this function will return the centrality score of the specified group. Thus the two functions can be used for two purposes: selecting key players or measuring group centrality.

The paper proceeds as follows. First, we review centrality measures at the individual level. Then we present methods for measuring centrality at the group level. After that, we present a greedy search algorithm for selecting key players and outline the basic structure and the usage of the main function kpset in package keyplayer. To illustrate the methods and the usage of the package, we use a simulated network as well as an empirical example based on the friendship network among managers in a company. Last, we summarize and point out directions for improving the package in the future.

\section{Measuring individual centrality}

We first review the definitions of centrality measures at the individual level. For conciseness, we provide the definitions based on weighted networks, where the weight of a tie takes a continuous value and usually measures the strength of the connection between two nodes. The definitions naturally incorporate binary networks where the weight of a tie can only be one or zero, indicating the presence or absence of a connection (Freeman, 1978; Wasserman and Faust, 1994; Butts, 2008a).

Figure 1 shows an example of a simulated network. On the left is the adjacency matrix of the network. On the right is the network graph. Thinking of it as a friendship network, we can see that the strength of friendship between node 1 and node 3 is conceived differently by node 1 and node 3 . The former assigns it a weight of 3 while the latter assigns it a weight of 1 . We will use this example to illustrate the centrality measures. Calculations of four centrality measures (i.e., degree, closeness, betweenness, and eigenvector centralities) at the individual level are done using the sna package (Butts, 2008a). Calculations of four other individual level centralities and all group level centralities are done using our package keyplayer. We would like to clarify at this point that our package does not depend on sna. We use sna here just for the sake of the example.

\section{Degree centrality}

Degree centrality is defined as follows (Freeman, 1978; Butts, 2008a): ${ }^{1}$

$$
D_{i}=\sum_{j} w_{i j}+\sum_{j} w_{j i}
$$

where $w_{i j}$ represents the tie status from node $i$ to node $j$. Thus the first term indicates the outgoing connections from node $i$ (i.e., outdegree) and the second term the incoming connections to node $i$ (i.e., indegree). Degree centrality measures a node's direct connectedness with other nodes in a network. After loading the adjacency matrix in R, we can type the following in $\mathrm{R}$ to get the degree, indegree, and outdegree measures for the simulated network.

\footnotetext{
${ }^{1}$ It may be worth noting that Freeman (1978) distinguishes absolute and relative measures of centrality. The definition here is based on Butts (2008a) and only considers the absolute number of connections.
} 


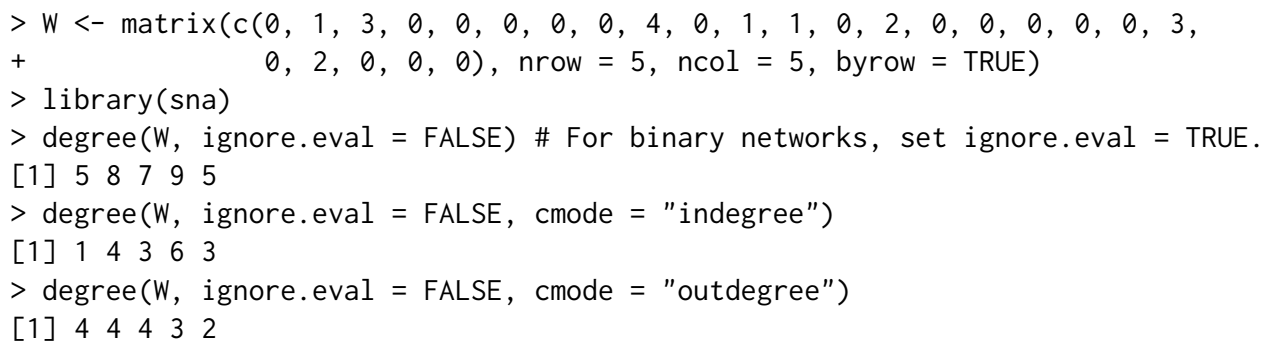

\section{Closeness centrality}

One version of the closeness centrality is due to Gil and Schmidt (1996):

$$
C_{i}=\frac{\sum_{j} d_{i j}^{-1}}{n-1}
$$

where $d_{i j}$ is the shortest path (i.e., geodistance) between nodes $i$ and $j$. Closeness centrality usually reflects a node's capability of quickly reaching other nodes. In the above example, the tie status indicates friendship strength. The larger the value, the stronger is the friendship. To have the shortest path correspond to the strongest friendship, we need to transform the tie status, for example, by taking the inverse, before calculating the closeness centrality.

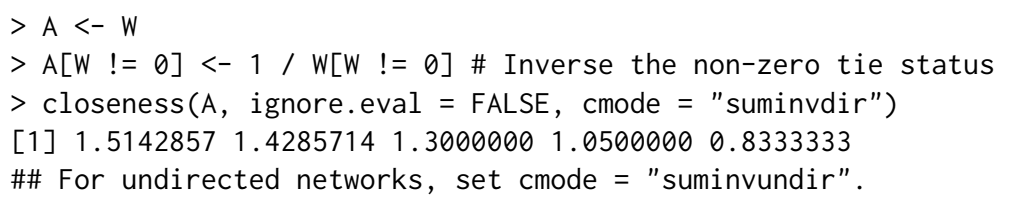

\section{Betweenness centrality}

Betweenness centrality is defined as follows (Butts, 2008a):

$$
B_{i}=\sum_{j k} \frac{g_{j k}^{i}}{g_{j k}},
$$

where $g_{j k}$ is the number of shortest paths between nodes $j$ and $k$, and $g_{j k}^{i}$ is the number of those paths that pass node $i$. In the case of $g_{j k}=0$, the corresponding contribution to the betweenness score is zero. Betweenness centrality usually measures a node's brokerage power in a network. We can get the betweenness centrality for the simulated network as follows.

$>$ betweenness (A, ignore.eval $=$ FALSE, cmode $=$ "directed")

[1] $0 \begin{array}{llllll}0 & 1 & 2 & 3 & 1\end{array}$

\section{Eigenvector centrality}

Eigenvector centrality defines a node's centrality as a weighted average of the centrality of its neighbors (Bonacich, 1972; Butts, 2008a):

$$
E_{i}=\frac{1}{\lambda} \sum_{j} w_{\mathrm{ij}} E_{j}
$$

In matrix notations, this is equivalent to

$$
\lambda E=W E,
$$

where $W$ represents the adjacency matrix and $\lambda$ the largest eigenvalue of the above equation. Eigenvector centrality measures the extent to which a node is connected to important alters. To get the eigenvector centrality for the adjacency matrix A, we type the following in R:

$>$ evcent $(A$, gmode $=$ "digraph", ignore.eval = FALSE, use.eigen = TRUE)

[1] $0.5000000+0 \mathrm{i} \quad 0.0000000+0 \mathrm{i} \quad 0.8660254+0 \mathrm{i} \quad 0.0000000+0 \mathrm{i} \quad 0.0000000+0 \mathrm{i}$

where gmode = "digraph" indicates that the input is a directed network and use. eigen = TRUE requests using the robust eigen function to calculate the eigenvectors. In this example, the eigenvector 
centrality includes complex numbers, which are hard to interpret. Thus, to facilitate interpretation of the results, it is often a good idea to symmetrize the network first because symmetric matrices always have real eigenvalues. In the following, the symmetrization process first converts $W$ to a binary matrix and then treats all ties as mutual ties.

$>\mathrm{B}<-$ symmetrize $(\mathrm{W})$

$>$ evcent $(B)$

[1] $0.3505418 \quad 0.5590326 \quad 0.4699593 \quad 0.4699593 \quad 0.3505418$

\section{M-reach degree centrality}

M-reach degree centrality generalizes the degree centrality by delimiting specific neighborhoods. Suppose the set of nodes that node $i$ can reach via $M$ steps is $F$ and the set of nodes that can reach node $i$ via $M$ steps is $H$. Building on Borgatti (2006), we define the M-reach centrality as follows:

$$
M_{i}=\sum_{j \in F} m_{i j}+\sum_{j \in H} m_{j i}
$$

where $m_{i j}$ is 1 if $j \in F$ and $m_{j i}$ is 1 if $j \in H$. The first term indicates the number of nodes that node $i$ can reach in $M$ steps. The second term indicates the number of nodes that can reach node $i$ in $M$ steps. By default, the matrix is binarized before calculating the centrality. Thus, in binary networks, the 1-reach degree centrality is the same as the degree centrality.

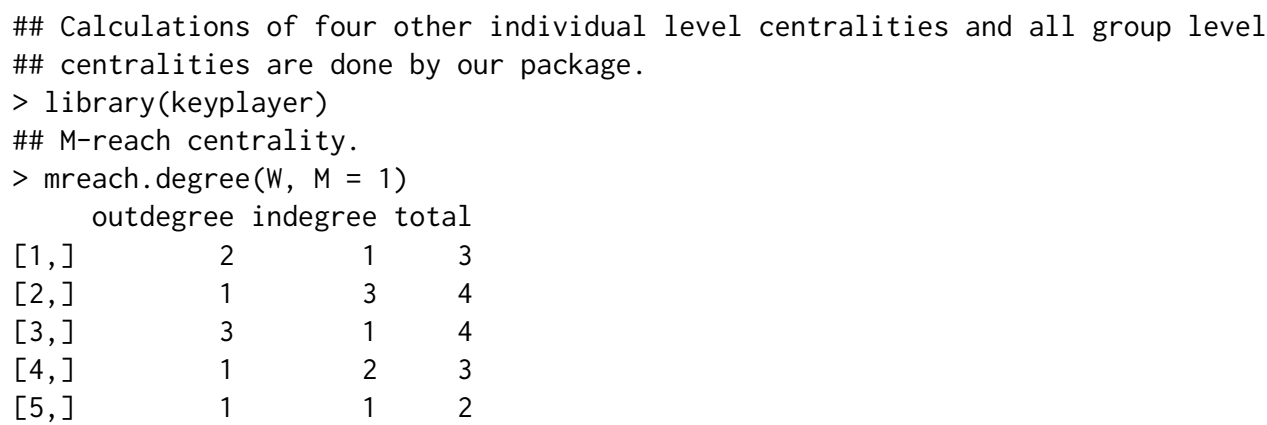

\section{M-reach closeness centrality}

One way to refine the M-reach degree centrality is to use (the inverse of) geodistance to measure the tie status between nodes, just like how closeness centrality refines degree centrality. We define the M-reach closeness centrality as below:

$$
M C_{i}=\frac{\sum_{j \in F} d_{i j}^{-1}}{d(n-1)}+\frac{\sum_{j \in H} d_{j i}^{-1}}{d(n-1)},
$$

where $d_{i j}$ is the geodistance between nodes $i$ and $j, F$ and $H$ are the set of nodes reachable from or to node $i$ via $M$ steps, respectively. $d$ is the maximal of $d_{i j}^{-1}$ across all pairs of $i$ and $j$. The denominator helps normalize each of the two terms to be between zero and one. When $M$ is infinity, M-reach closeness centrality approximates the Gil-Schmidt power index (Gil and Schmidt, 1996) and the cohesion centrality (Borgatti, 2006).

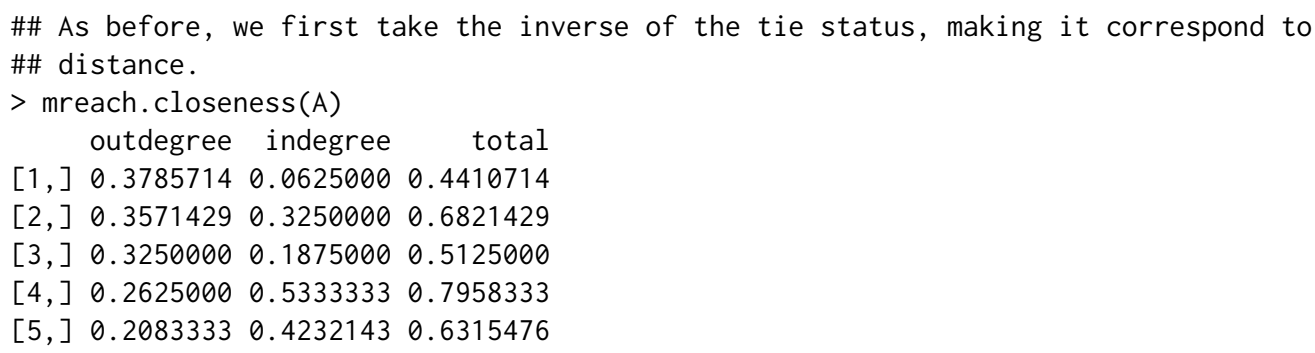




\section{Fragmentation centrality}

Fragmentation centrality measures the extent to which a network is fragmented after a node is removed from the network (Borgatti, 2006):

$$
F_{i}=1-\frac{\sum_{j, k \neq i} d_{j k}^{-1}}{d \cdot(n-1)(n-2)}
$$

where $d_{j k}$ is the geodistance between nodes $j$ and $k$ in the residual network after node $i$ is removed and $d$ the maximal of $d_{j k}^{-1}$ across $j$ and $k$. The second term in the above equation measures the cohesion of the residual network. Thus fragmentation centrality is the opposite of the cohesion centrality.
$>$ fragment $(A)$
fragment
$[1]$,
$[2]$,
$[3]$,
$[4]$,
$[5]$,

\section{Diffusion centrality}

Banerjee et al. (2013) proposed the diffusion centrality defined by the row sum of the following matrix:

$$
S=\sum_{t=1}^{T} P^{t},
$$

where $P$ is a probability matrix where $P_{i j}$ measures the probability that node $i$ can reach to node $j .{ }^{2}$ Each cell in the matrix $S$ measures the aggregate propensity that $i$ can reach to $j$ in $T$ iterations. Each row sum of the matrix $S$ indicates the importance of a node in disseminating information to alters (namely, the expected number of times that all alters receive the information from that node). Banerjee et al. (2014) show that as $T$ goes to infinity the diffusion centrality can approximate the eigenvector centrality or the Katz-Bonacich centrality (Katz 1953; Bonacich 1987). In practice, Banerjee et al. (2013) used the diffusion centrality to study the word-of-mouth information dissemination. Now suppose we create a new adjacency matrix by treating non-zero elements in the original network as ones and we also know what $q$ is. Then we can calculate the diffusion centrality as below.

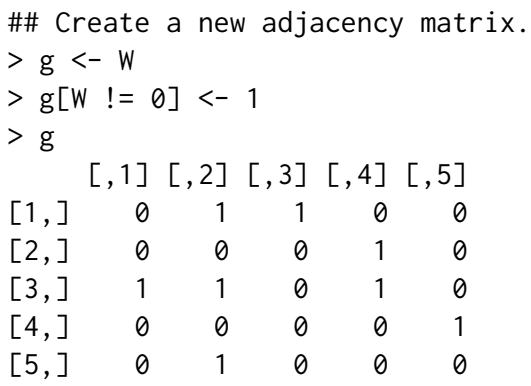

\#\# Create a matrix with the passing probabilities.

$>\mathrm{q}<-\operatorname{matrix}(\mathrm{c}(0, .2, .6,0,0, .1,0,0, .4,0, .1, .1,0, .4,0,0, .5,0,0, .3$,

$+$

$>\mathrm{q}$

$0, .4,0,0,0)$, nrow $=5$, ncol $=5$, byrow $=$ TRUE)

$[, 1][, 2][, 3][, 4][, 5]$

$\left[\begin{array}{llllll}1,] & 0.0 & 0.2 & 0.6 & 0.0 & 0.0\end{array}\right.$

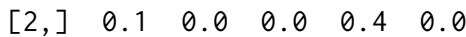

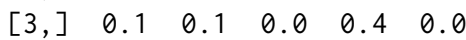

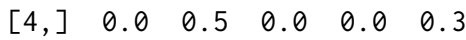

$\left[\begin{array}{llllll}5,] & 0.0 & 0.4 & 0.0 & 0.0 & 0.0\end{array}\right.$

\#\# Get the probability matrix and calculate diffusion centrality.

\footnotetext{
${ }^{2}$ In its original parametrization (Banerjee et al., 2013), $P=q \times g$, where $q$ is a measure of the passing probability and $g$ the adjacency matrix. For simplification and consistency with other centrality measures, our package asks users to input the probability matrix $P$ directly. With information on $q$ and the adjacency matrix, the probability matrix $P$ can easily be calculated by their product. Below we show an example of how to accomplish this.
} 


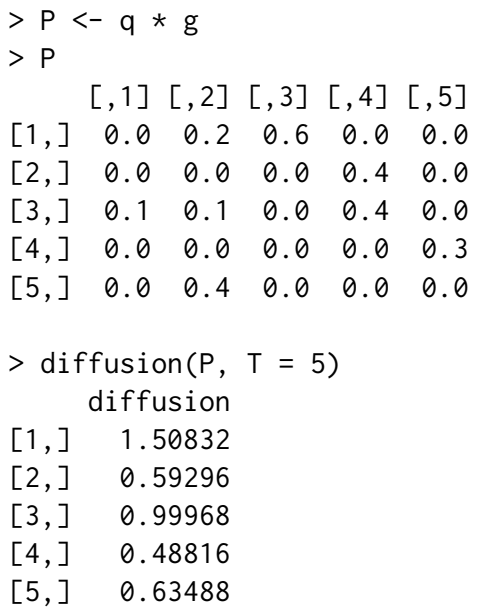

\section{Measuring group centrality}

Everett and Borgatti (1999) provide one of the first studies that explored ways to measure group centralities (mainly degree, closeness, and betweenness centralities) in undirected networks. In this paper, we provide more group centrality measures (including the eight ones outlined above) and extend the methods to both undirected and directed networks. The basic idea is to treat a group of nodes as a large pseudo-node. The key problem, then, is how to measure the tie status between the group and other outside nodes. For that purpose, we provide several criteria.

1. Minimum. According to the minimum criterion, the tie status between a group $G$ and an outside node $j$ is measured as the minimum of the (nonzero) edges between nodes in the group and the outside node.

$$
E_{G j}=\min _{g \in G} E_{g j} .
$$

This criterion ensures that there is a shortest path between the group and the outside node. It is useful for calculating geodistance related measures. Hence, by default we use this criterion to calculate the group level measures of geodistance, closeness centrality, betweenness centrality, M-reach centralities, and fragmentation centrality.

2. Maximum. According to the maximum criterion, the tie status between a group $G$ and an outside node $j$ is measured as the maximum of the (nonzero) edges between nodes in the group and the outside node.

$$
E_{G j}=\max _{g \in G} E_{g j} .
$$

This criterion is useful for measuring the maximal strength (not just the presence) of the connections between the group and the outside node. By default, we use the maximum criterion to compute the group level degree centrality and eigenvector centrality.

3. Addition. According to the addition criterion, the tie status between a group $G$ and an outside node $j$ is measured as the sum of the edges between nodes in the group and the outside node.

$$
E_{G j}=\sum_{g \in G} E_{g j}
$$

This criterion is useful for measuring the overall strength of the connections between the group and the outside node.

4. Union. The union criterion is designed for probability matrices. The tie status between a group $G$ and an outside node $j$ is measured as the probability that there is at least one path connecting the group with the outside node.

$$
E_{G j}=1-\prod_{g \in G}\left(1-E_{g j}\right) .
$$

By default, we use the union criterion to calculate the group level diffusion centrality.

In the simulated network, suppose nodes 2 and 3 are grouped together. The connection between this group and node 4 according to the maximum criterion is $E_{G 4}=4$. Suppose we use matrix $P$ as a probability network. Then the union criterion gives $E_{G 4}=1-(1-0.4) \times(1-0.4)=0.64$. The contract function automates these calculations and returns a reduced network matrix in which the node index will be re-ordered with the group as the last node. 
\#\# Group nodes 2 and 3 and measure the connections between the group and outside nodes \#\# using the maximum criterion.

$>\operatorname{contract}(W, c(2,3)$, method $=" \max ")$

145 set

$\begin{array}{lllll}1 & 0 & 0 & 0 & 3\end{array}$

$\begin{array}{lllll}4 & 0 & 0 & 3 & 0\end{array}$

$5 \quad 0 \quad 0 \quad 0 \quad 2$

set $1400 \quad 0$

\#\# Group nodes 2 and 3 in the probability matrix and measure the connections

\#\# between the group and outside nodes using the union criterion.

$>\operatorname{contract}(P, \mathrm{c}(2,3)$, method = "union")

$\begin{array}{llll}1 & 4 & 5 & \text { set }\end{array}$

$10.00 .00 \quad 0.0 \quad 0.68$

$4 \quad 0.0 \quad 0.00 \quad 0.3 \quad 0.00$

$\begin{array}{llllll}5 & 0.0 & 0.00 & 0.0 & 0.40\end{array}$

set $0.10 .64 \quad 0.0 \quad 0.00$

Once the tie status between the group and outside nodes is measured, we can use the centrality measures outlined above to calculate group centrality based on the reduced network. The kpcent function implements the calculations. Note that users do not need to explicitly deploy the contract function because kpcent automatically uses it in the background.

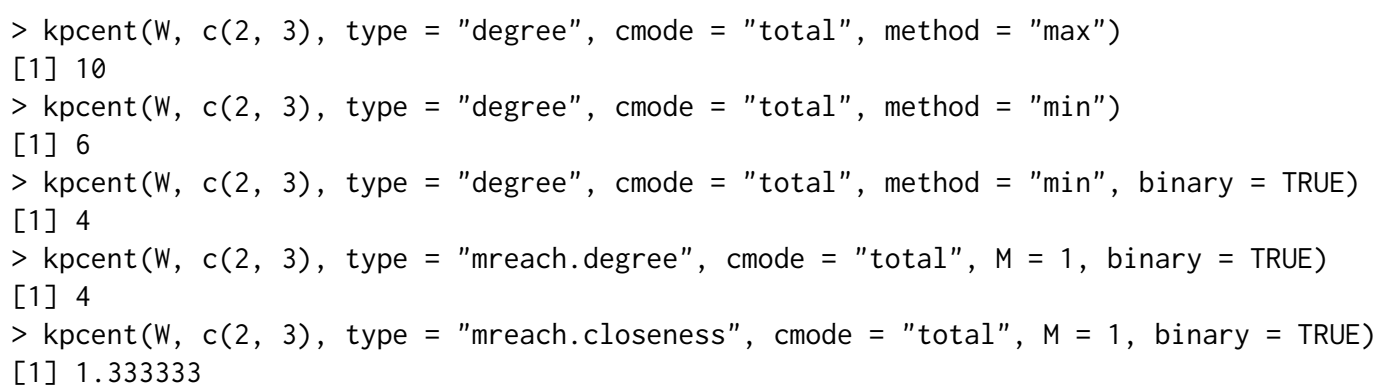

\section{Selecting key players}

Recall that the ultimate goal is to select the most central group of nodes from a network. This goal quickly becomes challenging as the network size grows. For example, to choose five key players out of 100 nodes, there are $\left(\begin{array}{c}100 \\ 5\end{array}\right)=75,287,520$ possible combinations. To search for the optimal set of key players, in keyplayer we employ a greedy search algorithm as originally proposed in Borgatti (2006). We revised the algorithm in multiple ways to enhance its usability and efficiency. The basic idea of the algorithm is to select a set of nodes as seeds and then swap the selected nodes with unselected ones if the swap increases the group centrality. More specifically, the algorithm proceeds as follows.

Step 1. Select an initial candidate set $C$. The residual set is denoted as $R$.

Step 2. Update the candidate set $C$.

1) Start with the first node in $C$. Try to swap it with nodes in $R$ sequentially (loop 1 ). Make the swap if it improves the centrality score of the resulting $C$. The number of iterations in loop 1 is defined as the number of iterations (over the nodes in the residual set).

2) Repeat loop 1 for each node in $C$ sequentially (loop 2). The number of iterations in loop 2 is defined as the number of rounds (over the nodes in the candidate set).

3) Stop if (1) the change in C's centrality score is smaller than a specified threshold or (2) the process reaches a specified number of rounds (i.e., the number of iterations in loop 2).

Step 3. Return the final set $C$ and the centrality score.

The function kpset implements the search algorithm. Its basic structure is shown below.

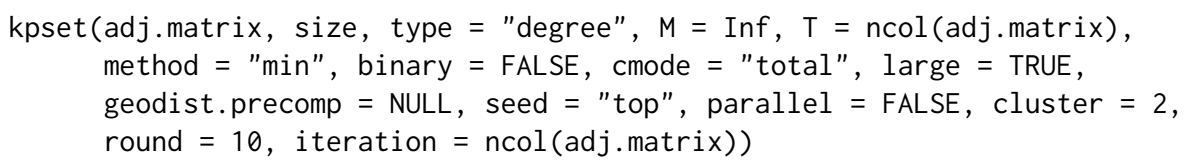

where the arguments are defined as follows. 
- adj. matrix: Matrix indicating the adjacency matrix of the network or in the case of diffusion centrality a probability matrix.

- size: Integer indicating the target size of players.

- type: String indicating the type of centrality measure to be used. Should be one of "degree" for degree centrality, "closeness" for closeness centrality, "betweenness" for betweenness centrality, "evcent" for eigenvector centrality, "mreach. degree" for M-reach degree centrality, "mreach.closeness" for M-reach closeness centrality, "fragment" for fragment centrality, and "diffusion" for diffusion centrality.

- M: Positive number indicating the maximum geodistance between two nodes, above which the two nodes are considered disconnected. The default is Inf. The option is applicable to M-reach degree, M-reach closeness, and fragmentation centralities.

- $\mathrm{T}$ : Integer indicating the maximum number of iterations in the communication process. For diffusion centrality only. By default, $\mathrm{T}$ is the network size.

- method: Indication of which grouping criterion should be used. "min" indicates the "minimum" criterion and is suggested for betweenness, closeness, fragmentation, and M-reach centralities. "max" indicates the "maximum" criterion and is suggested for degree and eigenvector centralities. "add" indicates the "addition" criterion and is suggested for degree and eigenvector centralities as an alternative of "max". "union" indicates the "union" criterion and is suggested for diffusion centrality. The default is "min".

- binary: If TRUE, the input matrix is binarized. If FALSE, the edge values are considered. The default is FALSE.

- cmode: String indicating the type of centrality being evaluated. The option is applicable to degree and M-reach centralities. "outdegree", "indegree", and "total" refer to indegree, outdegree, and total degree, respectively. "all" reports all the above measures. The default is to report the total degree.

- large: Logical scalar. If TRUE (the default), the method implemented in igraph is used for computing geodistance and related centrality measures; otherwise the method in sna is used.

- geodist.precomp: Geodistance precomputed for the network to be analyzed (optional).

- seed: String indicating the seeding method or a vector of the seeds specified by user. If "top", players with the highest individual centrality are used as the seeds. If "random", seeds are randomly sampled. The default is "top" for efficiency.

- parallel: Logical scalar. IF TRUE, the parallel computation is activated. The default is FALSE.

- cluster: Integer indicating the number of CPU cores to be used for parallel computation.

- round: Integer indicating the "length" of search, namely, the number of loops over the nodes in the candidate set.

- iteration: Integer indicating the "width" of search in each round, namely, the number of loops over the nodes in the residual set.

The greedy algorithm converges fast, but sometimes can be trapped in a local optimum. To avoid this problem, it is recommended to run kpset several times with different seeds. To facilitate the search in large networks, users can employ parallel computation by specifying parallel = TRUE in kpset. During parallel computation, for each cluster and each iteration the algorithm randomly picks a node from the candidate set and the residual set, respectively, and swaps the two if it improves the centrality score of the candidate set. It repeats this process until exhausting the specified iterations and rounds and then combines the results from the clusters. The following code shows how to find two players who are the most central as a group in the simulated network.

\#\# In terms of indegree.

$>\operatorname{kpset}(W$, size $=2$, type $=$ "degree", cmode $=$ "indegree", method $=$ "max")

\$keyplayers

[1] 34

\$centrality

[1] 7

\#\# In terms of indegree in the binarized network.

$>\operatorname{kpset}(\mathrm{W}$, size $=2$, type $=$ "degree", cmode $=$ "indegree", binary $=$ TRUE,

$+\quad \operatorname{method}=$ "max")

\$keyplayers

[1] 24 


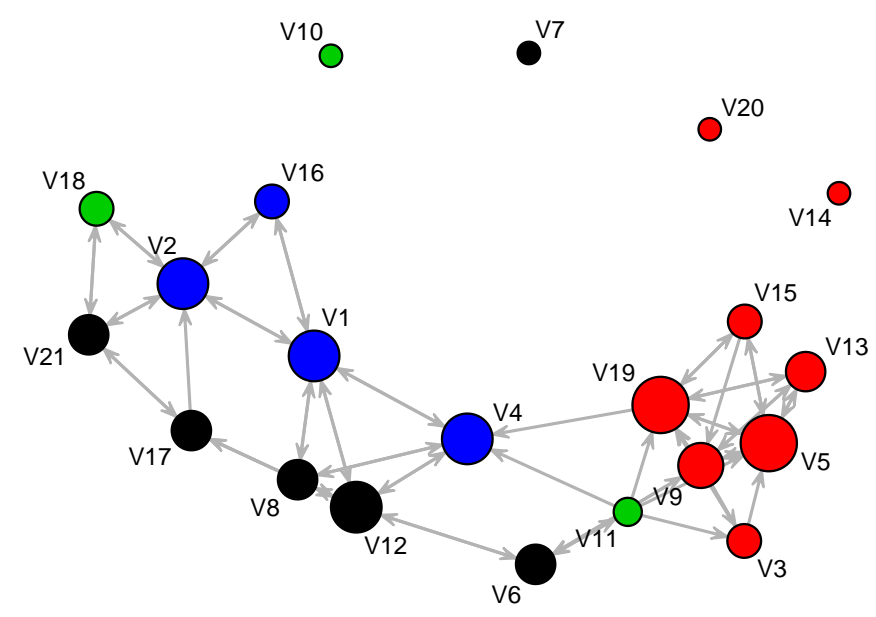

Figure 2: The friendship network of 21 managers in a high-tech company.

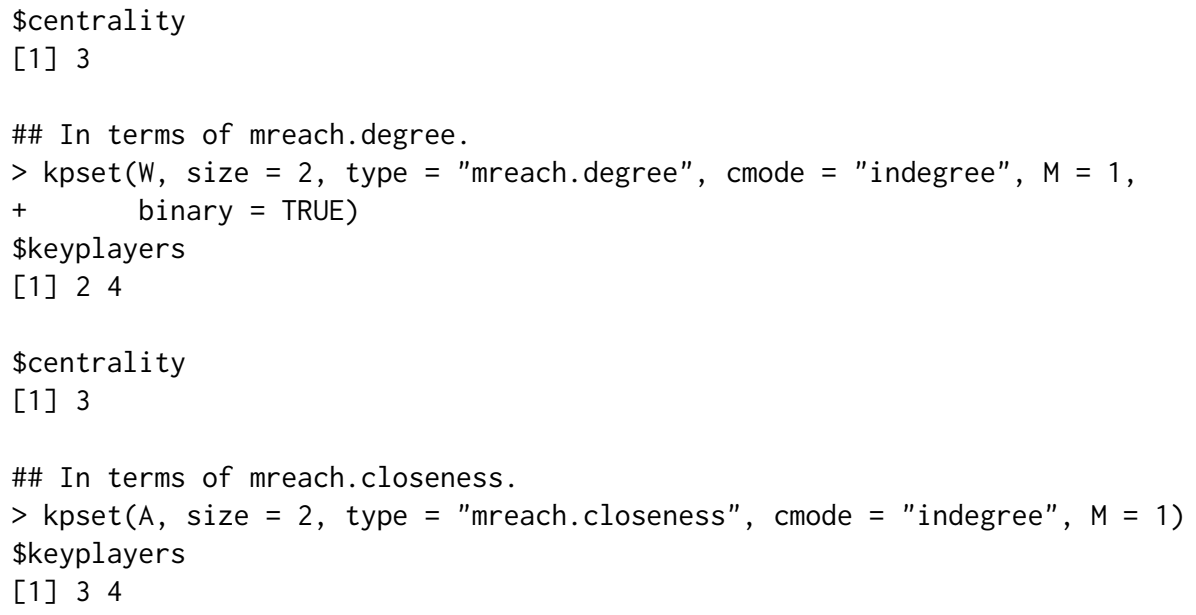

\section{An empirical example}

Below we use the friendship network of 21 managers in a high-tech company (Krackhardt, 1987) to illustrate the methods. The network graph is shown in Figure 2. Each node represents one manager. Each tie indicates a friendship nomination from one manager to the other. The nodes are colored according to the four departments the managers belong to. The size of each node is proportional to its degree. As it can be seen, friendships occur predominately within departments.

We first examine the individual centrality of the managers. To make a probability matrix for calculating the diffusion centrality, we multiply the original adjacency matrix by 0.1 . The results are presented in Table 1 . To facilitate reading the results, we marked the top centrality scores in red. Apparently, the most central manager identified varies with the centrality measure used. In terms of indegree, managers 5 and 19 each receive six friend nominations and are the most central. However, in terms of outdegree, managers 1, 9, 11, and 12 are the most central. In terms of closeness centrality, 


\begin{tabular}{|c|c|c|c|c|c|c|c|c|c|}
\hline$\overline{\mathrm{ID}}$ & Indegree & Outdegree & Closeness & Between & Evcent & $\begin{array}{r}\text { 2-reach } \\
\text { indegree }\end{array}$ & $\begin{array}{r}\text { 2-reach } \\
\text { closeness }\end{array}$ & Fragment. & $\begin{array}{r}\text { Diffusion } \\
(\mathrm{T}=2)\end{array}$ \\
\hline 1 & 5 & 5 & 0.44 & 60.83 & 0.32 & 11 & 0.40 & 0.71 & 0.68 \\
\hline 2 & 5 & 4 & 0.39 & 29.58 & 0.17 & 8 & 0.33 & 0.70 & 0.52 \\
\hline 3 & 2 & 2 & 0.34 & 0.00 & 0.14 & 6 & 0.20 & 0.68 & 0.28 \\
\hline 4 & 5 & 3 & 0.38 & 46.25 & 0.25 & 14 & 0.48 & 0.70 & 0.44 \\
\hline 5 & 6 & 4 & 0.40 & 13.00 & 0.28 & 7 & 0.33 & 0.69 & 0.55 \\
\hline 6 & 3 & 3 & 0.43 & 79.75 & 0.26 & 10 & 0.33 & 0.74 & 0.45 \\
\hline 7 & 0 & 0 & 0.00 & 0.00 & 0.00 & 0 & 0.00 & 0.65 & 0.00 \\
\hline 8 & 3 & 4 & 0.41 & 5.67 & 0.29 & 9 & 0.30 & 0.69 & 0.56 \\
\hline 9 & 4 & 5 & 0.45 & 47.08 & 0.31 & 8 & 0.30 & 0.70 & 0.66 \\
\hline 10 & 0 & 0 & 0.00 & 0.00 & 0.00 & 0 & 0.00 & 0.65 & 0.00 \\
\hline 11 & 1 & 5 & 0.48 & 20.50 & 0.32 & 3 & 0.10 & 0.69 & 0.66 \\
\hline 12 & 5 & 5 & 0.47 & 88.42 & 0.34 & 11 & 0.40 & 0.74 & 0.68 \\
\hline 13 & 3 & 3 & 0.37 & 1.17 & 0.23 & 7 & 0.25 & 0.68 & 0.43 \\
\hline 14 & 0 & 0 & 0.00 & 0.00 & 0.00 & 0 & 0.00 & 0.65 & 0.00 \\
\hline 15 & 2 & 3 & 0.37 & 1.17 & 0.23 & 6 & 0.20 & 0.68 & 0.43 \\
\hline 16 & 2 & 2 & 0.33 & 0.00 & 0.13 & 8 & 0.25 & 0.68 & 0.29 \\
\hline 17 & 3 & 3 & 0.39 & 27.83 & 0.17 & 8 & 0.28 & 0.69 & 0.42 \\
\hline 18 & 2 & 2 & 0.30 & 0.00 & 0.07 & 5 & 0.18 & 0.68 & 0.27 \\
\hline 19 & 6 & 4 & 0.42 & 44.17 & 0.26 & 7 & 0.33 & 0.70 & 0.53 \\
\hline 20 & 0 & 0 & 0.00 & 0.00 & 0.00 & 0 & 0.00 & 0.65 & 0.00 \\
\hline 21 & 3 & 3 & 0.35 & 8.58 & 0.11 & 7 & 0.25 & 0.68 & 0.39 \\
\hline
\end{tabular}

Table 1: Centrality scores for the managers.

\begin{tabular}{lrrrr}
\hline & KP1 & KP2 & KP3 & Score \\
\hline Indegree & 2 & 5 & 12 & 14.00 \\
Outdegree & 2 & 5 & 12 & 12.00 \\
Closeness & 1 & 9 & 21 & 0.72 \\
Betweenness & 2 & 12 & 19 & 130.17 \\
Evcent & 2 & 11 & 12 & 0.58 \\
2-reach indegree & 1 & 7 & 19 & 15.00 \\
2-reach closeness (indegree) & 2 & 5 & 12 & 0.78 \\
Fragmentation & 1 & 6 & 17 & 0.82 \\
Diffusion $(\mathrm{T}=2)$ & 1 & 9 & 11 & 1.93 \\
\hline
\end{tabular}

Table 2: The three managers who are the most central as a group.

manager 11 is the most central. In terms of betweenness centrality and eigenvector centrality, manager 12 is the most central, etc. Which centrality measure is suitable for selecting the most central player depends on the objectives. If the objective is to find a manager whose opinion is respected by most peers, then indegree can be a suitable measure. But if the objective is to spread the information most widely, then outdegree or closeness may be a better option.

Now suppose we want to find the three managers in this company who are the most central as a group. Table 2 lists the results according to different centrality measures. If indegree is the preferred centrality measure, then managers 2, 12, and 19 form the most central group. Together these three managers can connect to 14 other managers. Note that the three managers with the highest individual centrality do not constitute the most central group. The group indegree of managers 5, 19, and 1 (or 2 or 4 ) is no more than 10 . Table 2 also shows that the most central group varies by centrality measure employed. Researchers are required to thoroughly thinkabout which centrality measure they should use in their specific context to select key players. In addition, sometimes there may be multiple sets of players which are equally central as a group. In such cases, which set is to be used may not make big difference in practice. But if examining these different sets is of interest, it is recommended to run kpset multiple times.

\section{Summary}

In this paper, we developed a comprehensive set of methods and tools for locating key players in social networks. In the future, the algorithms used may be improved by choosing seeds and swaps more strategically and by utilizing alternative optimization schemes such as simulated annealing. 


\section{Acknowledgments}

The two authors contributed equally. Weihua An designed the study. Both authors contributed to writing the manuscript and developing the package. The authors thank Professor Bettina Grün and two anonymous reviewers for their helpful comments.

\section{Bibliography}

W. An. Multilevel meta network analysis with application to studying network dynamics of network interventions. Social Networks, 43:48-56, 2015. [p257]

W. An and Y.-H. Liu. keyplayer: Locating Key Players in Social Networks, 2016. URL https: //CRAN. Rproject.org/package=keyplayer. R package version 1.0.3. [p257]

A. Banerjee, A. G. Chandrasekhar, E. Duflo, and M. O. Jackson. Diffusion of microfinance. Science, 341 (6144):1-7, 2013. doi: 10.1126/science.1236498. [p257, 261]

A. Banerjee, A. G. Chandrasekhar, E. Duflo, and M. O. Jackson. Gossip: Identifying central individuals in a social network. Working Paper, 2014. [p261]

P. Bonacich. Factoring and weighting approaches to clique identification. Journal of Mathematical Sociology, 2(1):113-120, 1972. [p259]

P. Bonacich. Power and centrality: A family of measures. American Journal of Sociology, 5:1170-1182, 1987. [p261]

S. P. Borgatti. Identifying sets of key players in a network. Computational, Mathematical and Organizational Theory, 12:21-34, 2006. [p257, 260, 261, 263]

S. P. Borgatti, M. G. Everett, and L. C. Freeman. UCINET for Windows: Software for Social Network Analysis. Analytic Technologies, Harvard, MA, 2002. [p257]

R. S. Burt. Structural Holes: The Social Structure of Competition. Harvard University Press, Cambridge, MA, 1992. [p257]

C. T. Butts. Social network analysis with sna. Journal of Statistical Software, 24(6):1-51, 2008a. doi: 10.18637/jss.v024.i06. [p257, 258, 259]

C. T. Butts. network: A package for managing relational data in R. Journal of Statistical Software, 24(2): 1-36, 2008b. doi: 10.18637/jss.v024.i02. [p257]

R. Campbell, F. Starkey, J. Holliday, S. Audrey, M. Bloor, N. Parry-Langdon, R. Hughes, and L. Moore. An informal school-based peer-led intervention for smoking prevention in adolescence (ASSIST): A cluster randomised trial. Lancet, 371:1595-1602, 2008. [p257]

G. Csardi and T. Nepusz. The igraph software package for complex network research. InterJournal, Complex Systems:1695, 2006. URL http: //igraph.org. [p257]

M. G. Everett and S. P. Borgatti. The centrality of groups and classes. Journal of Mathematical Sociology, 23(3):181-201, 1999. [p262]

L. C. Freeman. Centrality in social networks: Conceptual clarification. Social Networks, 1:215-239, 1978. [p258]

J. Gil and S. Schmidt. The origin of the Mexican network of power. In Proceedings of the International Social Network Conference, pages 22-25, Charleston, SC, 1996. [p259, 260]

M. S. Handcock, D. R. Hunter, C. T. Butts, S. M. Goodreau, and M. Morris. statnet: Software tools for the representation, visualization, analysis and simulation of network data. Journal of Statistical Software, 24(1):1-11, 2008. doi: 10.18637/jss.v024.i01. [p257]

L. Katz. A new status index derived from sociometric index. Psychometrika, 18(1):39-43, 1953. [p261]

J. A. Kelly, S. L. Janet, E. D. Yolanda, L. Y. Stevenson, A. C. Hauth, T. L. Brasfiel, S. C. Kalichman, J. E. Smith, and M. E. Andrew. HIV risk behavior reduction following intervention with key opinion leaders of population: An experimental analysis. American Journal of Public Health, 81:168-171, 1991. [p257]

D. Krackhardt. Cognitive social structures. Social Networks, 9:109-134, 1987. [p265] 
C. A. Latkin. Outreach in natural settings: The use of peer leaders for HIV prevention among injecting drug users' networks. Public Health Reports, 113:S151-S159, 1998. [p257]

S. Ressler. Social network analysis as an approach to combat terrorism: Past, present, and future research. Homeland Security Affairs, 2(2):1-9, 2006. [p257]

R. Ripley, K. Boitmanis, and T. A. B. Snijders. RSiena: Siena - Simulation Investigation for Empirical Network Analysis, 2013. URL https://CRAN.R-project.org/package=RSiena. R package version 1.1-232. [p257]

J. Simon and K. Aditya. influenceR: Software Tools to Quantify Structural Importance of Nodes in a Network, 2015. URL https://CRAN. R-project.org/package=influenceR. R package version 0.1.0. [p257]

T. W. Valente and K. Fujimoto. Bridging: Locating critical connectors in a network. Social Networks, 2 (3):212-220, 2010. [p257]

T. W. Valente and P. Pumpuang. Identifying opinion leaders to promote behavior change. Health Education and Behavior, 34:881-896, 2007. [p257]

S. Wasserman and K. Faust. Social Network Analysis: Methods and Applications. Cambridge University Press, Cambridge, NY, 1994. [p258]

Weihua An

Departments of Statistics and Sociology, Indiana University

752 Ballantine Hall, 1020 East Kirkwood Avenue, Bloomington, IN 47405, USA.

weihuaan@indiana.edu

Yu-Hsin Liu

Kelley School of Business, Indiana University

Hodge Hall 329, 1309 E 10th St, Bloomington, IN 47405, USA

yuhsliu@indiana. edu 